\title{
A Bayesian approach to fitting Gibbs processes with temporal random effects
}

\author{
Ruth King ${ }^{1}$, Janine B. Illian ${ }^{1}$, Stuart E. King ${ }^{1}$, Glenna F. \\ Nightingale $^{1}$ and Ditte K. Hendrichsen ${ }^{2}$ \\ ${ }^{1}$ School of Mathematics and Statistics, University of St Andrews, \\ St Andrews, Fife, Scotland \\ ${ }^{2}$ Norwegian Institute For Nature Research, N-7047, Trondheim, \\ Norway \\ ruth@mcs.st-and.ac.uk
}

\begin{abstract}
We consider spatial point pattern data that have been observed repeatedly over a period of time in an inhomogeneous environment. Each spatial point pattern can be regarded as a "snapshot" of the underlying point process at a series of times. Thus, the number of points and corresponding locations of points differ for each snapshot. Each snapshot can be analysed independently, but in many cases, there may be little information in the data relating to model parameters, particularly parameters relating to the interaction between points. Thus, we develop an integrated approach, simultaneously analysing all snapshots within a single robust and consistent analysis. We assume that sufficient time has passed between observation dates so that the spatial point patterns can be regarded as independent replicates, given spatial covariates. We develop a joint mixed effects Gibbs point process model for the replicates of spatial point patterns by considering environmental covariates in the analysis as fixed effects, to model the heterogeneous environment, with a random effect (or hierarchical) component to account for the different observation days for the intensity function. We demonstrate how the model can be fitted within a Bayesian framework using an auxiliary variable approach to deal with the issue of the random effects component. We apply the methods to a dataset of muskoxen herds and demonstrate the increased precision of the parameter estimates when considering all available data within a single integrated analysis.
\end{abstract}

Keywords: Data augmentation; Markov chain Monte Carlo; Mixed effects model; Muskoxen data; Spatial and temporal point processes

\section{Introduction}

Collecting ecological data on the behaviour of individuals in space, i.e. data that detail the exact spatial location of individuals, can be rather costly and/or 
technically challenging. Recent technology facilitates the collection of spatially explicit data, for example via the use of GPS/Argos collars attached to individuals that record the location of the individual at given time intervals over a period of time until the end of the experiment or the device "fails" (for example from battery failure or the device becoming detached from the individual). Applications range from large terrestrial (Babin et al. 2011 and Langrock et al. 2012, bison; Morales et al. 2004, elk) and marine mammals (Johnson et al. 2008, harbour seal; Jonsen et al. 2005, McClintock et al. 2012, grey seal) to small insects (Wikelski et al. 2010, orchid bees) to name just a few. In such studies, the biological mechanisms of interest often relate to the movement of individuals over time and attributed to different underlying behaviours (Blackwell 2003, Morales et al. 2004, Breed et al. 2009 and McClintock et al. 2012). However, in such studies only very few individuals from the study population are typically monitored and the resulting data for individuals either analysed independently of each other or assuming a common underlying model. Recently, multiple animals have been considered simultaneously using hierarchical random effect models to allow for individual variability, yet still only a small proportion of all individuals are typically tagged (Langrock et al. 2012). Consequently, it is difficult to directly model interactions between individuals and draw meaningful biological conclusions relating to interactions between different individuals/groups.

Alternatively, using for example an aerial survey, a snapshot can be taken of the locations of all individuals within a given study area at a particular point in time, i.e. a spatial pattern formed by the individuals at a given time (Cornulier and Bretagnolle 2006, Illera et al. 2010). However, there is often limited information contained within a single point pattern for drawing robust conclusions on the ecological mechanisms of interest. This may lead to a series of point patterns collected at repeated points in time. The snapshots of the same biological species may be taken over different (non-overlapping) geographical regions or repeatedly over the same study region for mobile individuals (as we will consider in this paper). For such repeated point pattern data, individual points (i.e. individuals) are not uniquely identifiable (i.e. given any two individual points in different snapshots we cannot identify whether they are the same individual or not) so it is not possible to consider explicit spatio-temporal movement models similar to those developed for GPS data, since these require the time-series of the locations of individual animals. Thus, such snapshot data can be seen to be in direct contrast to GPS collar data in that snapshots provide the location of all individuals but only for a small number of times (possibly a single time); whereas GPS collar data typically provides a long time-series of data on only a small number of individuals (possibly only a single individual).

In general, for a single spatial point pattern dataset, the geometric arrangement of the points in space that would be observed in a given area depends on both the underlying intensity of individuals and the corresponding interaction amongst these. Individual animals typically move around their home-range, reacting to the environment and the location (or perceived location) of other individuals (Jetz et al. 2004). For example, predators will be attracted to the 
location of their prey, while prey will be repulsed from the location of their predators or animals may exhibit territorial behaviour. Plants may repel each other as they compete for the same available resources, so that plants may be unable to grow near another plant due to limited resources (one of the individual plants is unable to compete as effectively for the resources and dies). Alternatively, individuals may attract each other through mechanisms such as facilitation where a plant species provides nutrients for another species (Brooker et al. 2008, Illian et al. 2009). Individuals within a plant or animal population typically perceive only their local neighbours and hence predominantly interact with these (Law et al. 2001). Hence, the parameters in a Gibbs (or Markov) point process model reflect local spatial interactions as well as the underlying intensity of the points. Interactions can be regarded as "negative" or "positive" between points, corresponding to repulsion or attraction among individuals. Heterogeneity within the study area that may influence the local intensity and/or interaction within the pattern, may be modelled via the use of spatial covariate information. If covariate data are available, these are typically included in a Gibbs process model as a fixed effect, often by a parametric function of the given spatial covariate(s) (Baddeley and Turner 2000; Baddeley and Turner 2006).

We consider a series of spatial point pattern data observed over a period of time, rather than a single point pattern. In many applications the number of observed points in a single pattern may be too low for the resulting estimates to be reliable, with very poor precision of the parameter estimates. Alternatively, in other cases, even when it is possible to obtain estimates of the intensity and interaction parameters for each independent dataset it can lead to inconsistent parameter estimates among the different snapshots. This makes it more difficult to sensibly interpret the model parameters, particularly if there is a general interest in population dynamics across years rather than within the specific years. We present an integrated approach where we jointly model all the spatial point patterns within a single analysis.

Integrated data analyses, simultaneously analysing all available data within a single analysis, can provide improved parameter estimates in terms of increased precision since information can be "borrowed" across the different datasets. Such integrated analyses are becoming increasing popular in the area of statistical ecology but are less common for spatial point processes; see for example Besbeas et al. (2003), Brooks et al. (2004), Schaub et al. (2007), Gauthier et al. (2010), King et al. (2008), Besbeas et al. (2009), Reynolds et al. (2009), King et al. (2009), Cave et al. (2010), McCrea et al. (2010), Illian et al. (2012b) and references therein. For spatial point patterns, repeated data could occur at the temporal of geographical scale. For snapshots taken over different (nonoverlapping) geographical regions, the observed point patterns are assumed to be independent, given the underlying environment. Using repeated snapshot data of the same study area, assuming that the periods between observation times are sufficiently long, the observed point patterns may be assumed to be independent, given the environment within the study area. Considering an integrated analysis permits the incorporation of regional variability (for snapshots 
of different regions) or temporal variability (for repeated snapshots of the same region) within a consistent framework. For the muskoxen data described in Section 2, snapshots are taken on a weekly basis during the study period. To demonstrate the methodology we consider snapshots which correspond to a lag of (approximately) one year, removing any potential dependence between successive snapshots (conditional on spatial covariates) and the need to consider additional factors such as seasonal migration (see Section 2 for further discussion).

We follow Illian and Hendrichsen (2010) who propose a mixed effects model resulting from replicated spatial point patterns, allowing for a temporal random effect component within the model combined with a fixed effects environmental component. In particular, they re-express the conditional likelihood so that it is equivalent to the log-likelihood of independent weighted Poisson variables using the Berman-Turner device. Consequently, this permits the calculation of the MLEs of the parameters via the use of standard software for generalised linear mixed models (GLMMs). However, this model-fitting approach assumes independence among the points of a single snapshot within the log-likelihood expression, so that the standard errors of the parameters and resulting test statistics generated by the software cannot be interpreted directly.

In this paper, we provide a framework for fitting mixed effects models within a Bayesian setting, where we are able to estimate the uncertainty associated with the parameter estimates, for example, using posterior credible intervals. In Section 2 we introduce the data and provide the mathematical notation used throughout. In Section 3 we describe a mixed effects model, where there are both fixed effects, corresponding to the environmental covariate, and temporal random effects that may influence the intensity of the point process. In Section 4 we describe a Bayesian approach (using Markov chain Monte Carlo and data augmentation) that can be used to fit the mixed effect model. We present the corresponding results obtained for the given data in Section 5. Finally, we conclude with a discussion in Section 6.

\section{Data and Notation}

We consider the locations of muskoxen herds (Ovibos moschatus) in an area near the Zackenberg Research Station in Northeast Greenland $\left(74^{\circ} 30^{\prime} \mathrm{N}, 21^{\circ} 00^{\prime} \mathrm{W}\right)$. Data are collected as part of the Zackenberg monitoring programme, with the dual aim of investigating ecosystem functioning in a high Arctic Ecosystem, and monitoring ecosystem response to climatic change. The muskoxen data are recorded weekly through field surveys and represent near-total counts, aging and sexing of animals within the valley. During the summer, muskoxen migrate into the area from adjacent areas and the density of animals within the valley therefore increases from late spring to early autumn (Berg 2003). We consider the snapshot of the muskoxen taken in week 34 of each year from 1999 to 2007 , except for 2006 where we use the snapshot in week 33 as this was the final snapshot collected in that year. These weeks lie in the height of the summer, before 
the autumn rut. Considering snapshots of (approximately) one year apart, it seems reasonable to be able to assume that they are independent of each other, due to the very long time lag between snapshots. In addition, considering the snapshots taken at the same time of year reduces additional temporal variability due to seasonal affects, including seasonal migration. The data snapshots are presented in Figure 1, including the corresponding boundary of the study area, plotted with graded contours for the altitude of the region. Clearly, we can see from the figure that there appears to be substantial variability in the number of muskoxen herds observed in the study period over time, from a minimum of 16 herds in 1999 to a maximum of 54 herds in 2004. In addition, Figure 1 suggests that the location of the herds are negatively related to altitude (i.e. there are fewer herds at higher altitudes), which has been identified in previous analyses (Forchhammer et al. 2008). Thus, we include altitude as an environmental covariate in the analysis.

[Figure 1 about here.]

Notationally, we let $N_{t}$ denote the number of observed muskoxen at time $t=1, \ldots, T$, where $T=9$, corresponding to years 1999 to 2007 and $x_{t j}$ the location of herd $j=1, \ldots, N_{t}$ at time $t=1, \ldots, T$. For notational convenience, we let $\boldsymbol{x}_{t}=\left\{x_{t j}: j=1, \ldots, N_{t}\right\}$, so that $\boldsymbol{x}_{t}$ denotes the set of muskoxen herd locations at time $t=1, \ldots, T$. In other words $\boldsymbol{x}_{t}$ denotes the spatial point pattern data at time $t$. The muskoxen herds represented by the points observed at a given time are assumed to interact. However, since the muskoxen herds are motile, they only interact with muskoxen observed at the same time point and are assumed to be independent of muskoxen locations at all other time periods, i.e. $\boldsymbol{x}_{t_{1}}$ and $\boldsymbol{x}_{t_{2}}$ are independent for $t_{1} \neq t_{2}$ (conditional on the environmental covariate). Further we set $\boldsymbol{x}=\left\{\boldsymbol{x}_{t}: t=1, \ldots, T\right\}$, so that $\boldsymbol{x}$ denotes the set of all spatial point pattern data. We let $W$ denote the study region. Finally, for location $u \in W$, we let $y(u)$ denote the corresponding (normalised) altitude of location $u$, and set $\boldsymbol{y}=\{y(u): u \in W\}$. We note that in this example $W$ is constant over time, but more generally it is possible to allow the study region to vary over the time points. Similarly, while the covariate altitude does not vary among the time points it is straightforward to include time-varying covariates in the model.

\section{Models}

We assume that the point patterns $\boldsymbol{x}_{1}, \ldots, \boldsymbol{x}_{T}$ are independent (conditional on the environmental covariate) and model them using a Gibbs process (Stoyan et al. 1995; van Lieshout 2000; Møller and Waagepetersen 2007; Illian et al. 2008). In other words, we assume that at time $t$ the density and hence the conditional intensity of the process is a function of the intensity and interaction parameters. We initially describe the point process model for a single point pattern, before extending the ideas to include both fixed effects (relating to an 
environmental covariate) and random effects (relating to temporal heterogeneity).

\subsection{Area interaction process}

We note that many Gibbs processes, in particular pair-wise interaction processes, may only be used to model repulsive inter-individual interaction. Animals that display scramble competition, i.e. utilize resources which they do not have exclusive access to, face a degree of cost associated with social interactions over access to forage and other resources, which may lead to such a repulsive interaction (Nicholson 1954; Hassell 1975). However, conversely, several factors may cause animals to aggregate, including kinship, mating behaviour and predator avoidance (Caraco et al. 1980; Janson 1988). Ungulates can exhibit both attraction and repulsion towards each other depending on factors such as the age, sex and breeding status (Li et al. 2012), so that we use an area interaction process (Baddeley and van Lieshout 1995) to potentially consider both positive and negative interactions for the muskoxen herds.

We initially consider a single spatial point pattern $\boldsymbol{x}_{t}=\left\{x_{t 1}, \ldots, x_{t N_{t}}\right\}$. The conditional intensity is given by,

$$
\lambda\left(x_{t j} ; \boldsymbol{x}_{t}\right)=\kappa_{t} \gamma_{t}^{-A_{W, t}\left(x_{t j}\right)},
$$

where,

$$
A_{W, t}\left(x_{t j}\right)=\mathcal{A}_{W, t}\left(\boldsymbol{x}_{t}\right)-\mathcal{A}_{W, t}\left(\boldsymbol{x}_{t} \backslash\left\{x_{t j}\right\}\right)
$$

such that $\mathcal{A}_{W, t}\left(\boldsymbol{x}_{t}\right)$ denotes the area in the region $W$ that lies within distance $R$ of any of the points in $\boldsymbol{x}_{t}=\left\{x_{t 1}, \ldots, x_{t N_{t}}\right\}$. Mathematically, if we let $D\left(x_{t j}\right)$ denote the disc of radius $R$ centred at $x_{t j}$ within region $W$, we can write $\mathcal{A}_{W, t}\left(\boldsymbol{x}_{t}\right)=\cup_{j=1}^{N_{t}} D\left(x_{t j}\right)$. Thus, $A_{W, t}\left(x_{t j}\right)$ is the area of "single occupancy" of the disc $D\left(x_{t j}\right)$ within region $W$ (i.e. the area of the disc of radius $R$ centred at $x_{t j}$ within region $W$ that does not overlap with any other discs of radius $R$ centred at $x_{t i}$, for all $i \neq j$ ).

We follow the approach used in $R$-library spatstat (Baddeley and Turner 2011) and use the transformation to the canonical scale-free parametrisation by setting $\eta_{t}=\gamma_{t}^{\pi R^{2}}$ and $\beta_{t}=\frac{\kappa_{t}}{\eta_{t}}$. Thus, the conditional intensity can be written as

$$
\lambda\left(x_{t j} ; \boldsymbol{x}_{t}\right)=\beta_{t} \eta_{t}^{1-B_{W, t}\left(x_{t j}\right)},
$$

where $B_{W, t}\left(x_{t j}\right)=A_{W, t}\left(x_{t j}\right) / \pi R^{2}$ and $1-B_{W, t}\left(x_{t j}\right)$ can be interpreted as the proportion of the disc centred at $x_{t j}$ that overlaps with the union of all other discs centred at $x_{t i}$ for $i \neq j$ within region $W$, or the proportion of the area of "multiple occupancy" for point $x_{t j}$ with respect to $\boldsymbol{x}_{t} \backslash x_{t j}$. The parameter $\beta_{t}$ is the intensity of the process and the parameter $\eta_{t}$ relates to the interaction strength of the process at time $t$. The pseudolikelihood can then be written as a product over all observed data points of the corresponding conditional intensity for each point (Besag 1977). For example, for the point process at time $t$, the 
pseudolikelihood is given by,

$$
\begin{aligned}
f\left(\boldsymbol{x}_{t} \mid \beta_{t}, \eta_{t}\right) & =\alpha\left(\beta_{t}, \eta_{t}\right) \prod_{j=1}^{N_{t}} \lambda\left(x_{t j} ; \boldsymbol{x}_{t}\right) \\
& =\alpha\left(\beta_{t}, \eta_{t}\right) \beta_{t}^{N_{t}} \eta_{t}^{\left(N_{t}-B_{W, t}\left(\boldsymbol{x}_{t}\right)\right)},
\end{aligned}
$$

where $B_{W, t}\left(\boldsymbol{x}_{t}\right)=\sum_{j=1}^{N_{t}} B_{W, t}\left(x_{t j}\right)$. The term $\alpha\left(\beta_{t}, \eta_{t}\right)$ is given by,

$$
\alpha\left(\beta_{t}, \eta_{t}\right)=\exp \left(-\int_{W} \lambda\left(u ; \boldsymbol{x}_{t}\right) d u\right),
$$

such that for $u \notin \boldsymbol{x}_{t}$,

$$
\lambda\left(u ; \boldsymbol{x}_{t}\right)=\beta_{t} \eta_{t}^{\left(1-B_{W, t}(u)\right)},
$$

where $B_{W, t}(u)=A_{W, t}(u) / \pi R^{2}=\left[\mathcal{A}_{W, t}\left(\boldsymbol{x}_{t} \cup\{u\}\right)-\mathcal{A}_{W, t}\left(\boldsymbol{x}_{t}\right)\right] / \pi R^{2}$.

It is possible to fit separate area interaction processes to each dataset $\boldsymbol{x}_{t}$ independently of the other datasets. However, we now extend these processes to allow for the intensity to be a function of the environmental covariates (in this case using altitude as an example) with a random effects component to model the temporal heterogeneity, allowing information to be shared across the different datasets.

\subsection{Mixed effects model}

We consider, in turn, the intensity and interaction functions within the model. For the intensity function, we assume a mixed effects model consisting of a fixed effects component relating to the environmental covariate (altitude) and a random effects (or hierarchical) component to model temporal heterogeneity. We initially assume that the interaction function, modelling the interaction among herds, is constant over time and space, but extend to a random effects model for the interaction function in Section 5.

We begin with the (more complex) intensity function. For $t=1, \ldots, T$, we specify the intensity function, evaluated at location $u \in W$ to be of log-linear form,

$$
\beta_{t}(u)=\exp \left(\theta_{1}+\phi_{1, t}+\delta \times y(u)\right),
$$

so that the intensity parameters are a function of location, and set

$$
\phi_{1, t} \sim N\left(0, \sigma^{2}\right),
$$

for $t=1, \ldots, T$. The term $\theta_{1}$ denotes the underlying intensity rate; $\phi_{1}=$ $\left\{\phi_{1, t}: t=1, \ldots, T\right\}$ the temporal random effects on the intensity rate with random effect variance term, $\sigma^{2}$, and $\delta$ the (homogeneous) fixed effect regression coefficient for the environmental covariate. We use normalised values for $y(u)$ throughout (normalised over the full region $W$ ) as we consider a Bayesian approach (see for example King et al. 2009, for further discussion). 
We now consider the interaction function. We assume that the interaction strength among the muskoxen herds is constant over time and study area. Thus, we set $\eta_{t}=\eta$ for all $t=1, \ldots, T$, and reparameterise to set $\theta_{2}=\log \eta$. Within this model a value of $\theta_{2}>0$ corresponds to attraction among the herds, while $\theta_{2}<0$ corresponds to inhibition.

\subsection{Likelihood}

The model parameters to be estimated are $\zeta=\left\{\theta_{1}, \delta, \sigma^{2}, \theta_{2}\right\}$. We initially consider the contribution to the likelihood for a single snapshot $\boldsymbol{x}_{t}$, extending equation (1). The intensity parameter is a function of the observed (normalised) environmental covariates $\boldsymbol{y}$ and random effect component. Substituting the interaction and intensity functions into equation (1) and integrating out the random effect term $\phi_{1, t}$, we obtain the likelihood contribution for snapshot $t$,

$f\left(\boldsymbol{x}_{t} \mid \boldsymbol{\zeta}, \boldsymbol{y}\right)=\int_{\mathbb{R}}\left[\alpha_{t}(\boldsymbol{\zeta}) \exp \left(\theta_{2} \times\left[N_{t}-B_{W, t}\left(\boldsymbol{x}_{t}\right)\right]\right) \prod_{j=1}^{N_{t}} \exp \left(\theta_{1}+\phi_{1, t}+\delta \times y\left(x_{t j}\right)\right) p\left(\phi_{1, t} \mid \sigma^{2}\right)\right] d \phi_{1, t}$,

where $p\left(\phi_{1, t} \mid \sigma^{2}\right)$ denotes the probability density function of a $N\left(0, \sigma^{2}\right)$ distribution evaluated at $\phi_{1, t}$ and,

$$
\alpha_{t}(\boldsymbol{\zeta})=\exp \left(-\int_{W} \exp \left(\theta_{2} \times\left[1-B_{W, t}(u)\right]\right) \exp \left(\theta_{1}+\phi_{1, t}+\delta \times y(u)\right) d u\right) .
$$

We note that the function $B_{W, t}(\cdot)$ has no closed mathematical form. To evaluate this function at $u$, say, we use a Monte Carlo type approach, randomly simulating a set of points in $D(u)$, the disc centred at $u$ within the region $W$, and calculate the proportion of points that fall in an area of "multiple occupancy" (i.e. within a distance of $R$ from any point in $\boldsymbol{x}_{t} \backslash\{u\}$ ). The integral within the function for the $\alpha$ term is analytically intractable, and is calculated via numerical integration. Note that typically the Berman-Turner device (Berman and Turner 1992; Baddeley and Turner 2000) is used to perform this numerical integration in the term $\alpha_{t}(\boldsymbol{\zeta})$ due to the simplification of the likelihood expression, but in general, alternative numerical integration algorithms can be used.

We assume that the spatial point patterns for different times are independent conditional on the environmental covariates, so that we can express the joint pseudolikelihood over all snapshots, in the form,

$$
f(\boldsymbol{x} \mid \boldsymbol{\zeta}, \boldsymbol{y})=\prod_{t=1}^{T} f\left(\boldsymbol{x}_{t} \mid \boldsymbol{\zeta}, \boldsymbol{y}\right)
$$

However, we note that we relax the independence assumption of the random effect terms in Section 5.3. 


\section{Methods}

We consider a Bayesian analysis of the data and initially describe the Markov chain Monte Carlo (MCMC) approach that we implement to obtain a sample from the posterior distribution of the model parameters of interest. To reduce the numerical integration necessary in the evaluation of the likelihood expression, we use a data augmentation approach (Tanner and Wong 1987). In particular we introduce the $\phi_{1}$ terms as auxiliary variables (or parameters) to be estimated within the Bayesian analysis. We then form the joint posterior distribution over the model parameters, $\boldsymbol{\zeta}$, and auxiliary variables, $\phi$, given by,

$$
\pi(\boldsymbol{\zeta}, \boldsymbol{\phi} \mid \boldsymbol{x}, \boldsymbol{y}) \propto f(\boldsymbol{x} \mid \boldsymbol{\zeta}, \boldsymbol{\phi}, \boldsymbol{y}) p(\boldsymbol{\zeta}, \boldsymbol{\phi}) .
$$

We briefly discuss the prior $p(\boldsymbol{\zeta}, \boldsymbol{\phi})$, before considering the (conditional) likelihood term $f(\boldsymbol{x} \mid \boldsymbol{\zeta}, \boldsymbol{\phi}, \boldsymbol{y})$. The prior on the parameters can be decomposed into,

$$
p(\boldsymbol{\zeta}, \boldsymbol{\phi})=p(\boldsymbol{\zeta}) p\left(\boldsymbol{\phi} \mid \sigma^{2}\right) .
$$

Assuming independence of the temporal random effects, we can write,

$$
p\left(\phi \mid \sigma^{2}\right)=\prod_{t=1}^{T} p\left(\phi_{1, t} \mid \sigma^{2}\right)
$$

where $p\left(\phi_{1, t} \mid \sigma^{2}\right)$ denotes the density of a Normal distribution with mean 0 and variance $\sigma^{2}$, corresponding to the temporal random effect component. We specify independent priors on the model parameters of the form,

$$
\begin{aligned}
\theta_{i} \sim N\left(\mu_{i}, \tau_{i}\right) & i & =1,2 ; \\
\sigma^{2} \sim \Gamma^{-1}\left(a_{1}, a_{2}\right) ; & & \delta N\left(\mu_{\delta}, \tau_{\delta}\right) .
\end{aligned}
$$

With no expert prior information on these parameters, we set $\mu_{1}=\mu_{2}=\mu_{\delta}=0$; $\tau_{1}=\tau_{2}=\tau_{\delta}=100^{2}$ and $a_{1}=a_{2}=0.001$. Note that we perform a prior sensitivity analysis by considering different prior specifications on the parameters (see Section 5 for further discussion).

We now return to consider the term $f\left(\boldsymbol{x} \mid \boldsymbol{\zeta}, \boldsymbol{\phi}_{1}, \boldsymbol{y}\right)$, which can be regarded as the conditional pseudolikelihood of the data, given both the model parameters, $\boldsymbol{\zeta}$, and random effect terms, $\phi_{1}$. This (conditional) pseudolikelihood can be explicitly written as,

$$
f\left(\boldsymbol{x} \mid \boldsymbol{\zeta}, \phi_{1}, \boldsymbol{y}\right)=\prod_{t=1}^{T} f_{t}\left(\boldsymbol{x}_{t} \mid \boldsymbol{\zeta}, \boldsymbol{\phi}_{1}, \boldsymbol{y}\right)
$$

where

$f_{t}\left(\boldsymbol{x}_{t} \mid \boldsymbol{\zeta}, \phi_{1}, \boldsymbol{y}\right)=\alpha_{t}(\boldsymbol{\zeta}) \exp \left(\theta_{2} \times\left[N_{t}-B_{W, t}\left(\boldsymbol{x}_{t}\right)\right]\right) \prod_{j=1}^{N_{t}} \exp \left(\theta_{1}+\phi_{1, t}+\delta \times y\left(x_{t j}\right)\right)$ 
where $\alpha_{t}(\boldsymbol{\zeta})$ is given in equation (3). The posterior distribution of the model parameters, $\boldsymbol{\zeta}$, can then be obtained by taking the marginal posterior distribution (i.e. integrating out over the random effects terms, $\phi_{1}$ ),

$$
\pi(\boldsymbol{\zeta} \mid \boldsymbol{x}, \boldsymbol{y})=\int \pi\left(\boldsymbol{\zeta}, \boldsymbol{\phi}_{1} \mid \boldsymbol{x}, \boldsymbol{y}\right) d \boldsymbol{\phi}_{1} .
$$

Thus, the Bayesian approach does not remove the necessity of integrating out the random effect terms, but the integration is essentially performed within the MCMC algorithm, which obtains a sample from the joint posterior distribution over both $\zeta$ and $\phi$. Given a sample from the joint posterior distribution, to obtain a sample from the marginal distribution of $\boldsymbol{\zeta}$ (i.e. to integrate out over $\phi$ ), we simply take the sampled $\zeta$ values, irrespective of the associated values of $\phi$ and calculate the corresponding summary statistics of interest (as we would when simply calculating marginal posterior summary statistics of a single parameter in any Bayesian analysis). See King et al. (2009) and Bonner et al. (2010) for further discussion.

To obtain a sample from the joint posterior distribution $\pi(\boldsymbol{\zeta}, \boldsymbol{\phi} \mid \boldsymbol{x}, \boldsymbol{y})$ we use a hybrid or Metropolis-within-Gibbs algorithm. In particular, at each iteration of the Markov chain, we cycle through each parameter and update the parameter using either a random walk Metropolis-Hastings step with Normal proposal density or Gibbs step (if the posterior conditional distribution is of standard form), see for example Brooks (1998) for further details. In particular, we use a Gibbs step for $\sigma^{2}$ (since the posterior conditional distribution has an Inverse Gamma distribution) and a Metropolis-Hastings step for all other parameters. To obtain efficient proposal updates for the Metropolis-Hastings step we used a pilot-tuning procedure that involved running a series of short MCMC simulations using a variety of different Normal proposal variances and comparing the mixing of the subsequent chains via a combination of trace plots, mean acceptance probabilities and autocorrelation function (acf) plots. To obtain a sample from the marginal distribution $\pi\left(\boldsymbol{\zeta}, \boldsymbol{\sigma}^{2} \mid \boldsymbol{x}, \boldsymbol{y}\right)$, we simply consider only those realisations from the MCMC algorithm for the model parameters $\zeta$ (essentially integrating out over the random effect terms). However, we note that using this approach we are also able to immediately provide posterior marginal estimates of the random effect parameters, which may be of interest themselves, as they provide information on the magnitude and/or sign of the random effect at each time.

For the particular datasets that we consider, the MCMC simulations are run for 10,000 iterations for each individual snapshot and 20,000 iterations for the integrated analysis, simultaneously analysing all datasets within a single analysis, with the first $10 \%$ discarded as burn-in in each case. The MetropolisHastings variance proposal parameter is pilot-tuned for each individual dataset. Independent replications starting from over-dispersed starting points produced essentially identical results and the Brooks-Gelman-Rubin (BGR) statistic for each of the parameters did not indicate any lack of convergence (Brooks and Gelman 1998). 


\section{Results}

We initially analyse each individual spatial point pattern separately. We then consider an integrated analysis, simultaneously analysing all available data, using random effects to model temporal heterogeneity, providing a consistent and robust approach. We set an interaction radius for the discs centred on each point to be $R=125$. This is slightly larger than the interaction radius used by Illian and Hendrichsen (2010), who use $2 R=200$ and consider a pairwise interaction function. The specification of the slightly larger radius here is to ensure there are observed overlaps between at least two herds within each dataset.

\subsection{Independent analysis}

We analyse each spatial point pattern independently for each year from 1999 to 2007 , so that we have parameters $\theta_{1, t}, \theta_{2, t}$ and $\delta_{t}$ for $t=1, \ldots, 9$. The corresponding MLEs are presented in Table 1(a) and the posterior median (standard deviation) and 95\% symmetric credible interval (CI) using the Bayesian analysis in Table 1(b). We use the posterior median as the marginal posterior distributions for the interaction parameter $\theta_{2}$ is skewed in a number of years. The posterior medians appear to be generally similar to the corresponding MLEs of the parameters (differences are greatest for the most heavily skewed posterior marginal distributions). Clearly, there appears to be significant variability in estimates of the intensity parameter $\theta_{1, t}$ over time (with several non-overlapping credible intervals between years). The posterior estimates of the interaction parameters $\theta_{2, t}$ have relatively low precision (high posterior standard deviation and wide $95 \%$ symmetric CIs), suggesting that, in general, there is limited information in a single spatial point pattern to estimate the interaction parameter. However, there is possibly some evidence that the interaction also varies over time, with some non-overlapping credible intervals with regard to $\theta_{2, t}$, and the value of 0 not contained within three $95 \%$ symmetric CIs (corresponding to no interaction present). The regression coefficient $\delta$ appears to be fairly similar across years, allowing for the uncertainty with regard to the estimate. For example, the $95 \%$ symmetric CIs for $\delta$ all overlap for the different years. The years with smaller numbers of herds observed (1999-2003, with a range of 16-25 herds) have generally wider credible intervals on the parameter estimates than years where larger numbers of herds are observed (2004-2007, with a range of 40-54 herds). This is to be expected, since the larger the number of data points observed within a single snapshot, the greater the amount of information in the dataset regarding the parameters. One would normally not attempt to fit a model to patterns as small as those for the earlier years, as there is not enough information in the data of a single pattern for inference.

[Table 1 about here.]

However, combining all datasets within a single integrated analysis using a mixed effects model allows information to be "borrowed" across snapshots, thus providing an integrated and robust analysis of all available data. Note that 
within the model described in Section 3 we initially assume the same interaction function and spatial environmental covariate dependence (i.e. $\theta_{2}$ and $\delta$ ) for each dataset, but allow for additional temporal variability in the intensity function via the additional random effects component, as described in Section 3.2.

\subsection{Integrated analyses}

We initially consider a mixed effects model for the intensity function and fixed effects (time homogeneous) model for the interaction function. The corresponding posterior summary statistics for the parameters are provided in Table 2. Clearly, the environmental covariate (altitude) has a negative influence on the intensity of the spatial point pattern (posterior probability of 1.00 that the influence is negative). In addition, there appears to be a fairly strong temporal effect on the underlying intensity rate of the spatial point patterns, with a posterior median of 0.27 for the random effect variance and $95 \%$ symmetric CI (0.09, 1.06). The random effect terms $\phi$ for the intensity function can be estimated directly, as they are imputed within the MCMC simulations. Given the presence of a temporal random effect, there is positive evidence (Bayes factor $>3$ ) for a negative temporal random effect compared to the underlying mean intensity rate in 2003 and strong evidence (Bayes factor > 20) in years 1999-2001 (Kass and Raftery 1995). Similarly, there is positive evidence of a positive temporal random effect in 2005 and 2006, and strong evidence in years 2004 and 2007. The posterior probability of a positive interaction (i.e. $\theta_{2}>0$ ) is equal to 0.87 (or Bayes factor of 6.8) providing positive evidence of clustering of the herds.

[Table 2 about here.]

These results appear to reflect the increase in population size between 1999 and 2007 reported by Hansen et al. (2011). During this period the population density in the valley (measured as the maximum number of animals observed from a fixed point on any given day during the summer census) increased from (approximately) 20 individuals in 1999 to 110 in 2007, after which it has started to decrease again (Hansen et al. 2011). The population in the Zackenberg region is part of a larger population spanning Wollaston Foreland, Clavering Island and adjacent regions, so that the population increase does not reflect changes in population size per se, but the net influx of animals into the Zackenberg valley during the summer season. This influx is likely to be caused by a higher than average forage availability and/or quality (Forchhammer et al. 2005), an assumption supported by the seasonal increase in calves, yearlings and females (Berg 2003; Hansen et al. 2011), i.e. animals with need for high quality forage.

Comparing the integrated results with those obtained from the separate analyses in Table 1, we can clearly see that the posterior precision of the parameters $\left(\theta_{1}, \delta\right.$ and $\left.\theta_{2}\right)$ is significantly improved within the integrated analysis. This is a direct result of using all available data within a single integrated analysis, allowing information to be shared across datasets. For example, the estimate of $\delta$ is more precise within the integrated analysis, and consequently clearly confirms a 
negative effect of altitude on intensity (i.e. fewer herds at higher altitudes). In the single analyses for each year, there is greater uncertainty in the magnitude of the effect of altitude on the intensity and even the sign of this parameter in years 2001 and 2005. In addition, within the independent analyses, the sign of the interaction parameter, $\theta_{2}$, corresponding to attraction/repulsion is generally unclear. However, within the integrated analysis, there is positive evidence that the interaction is positive, corresponding to clustering of muskoxen herds which is likely to be related to the spatial distribution of food resources causing herds to aggregate in areas with access to forage. In particular, there is considerable variation in the distribution of plant communities within the census area (Elberling et al. 2008) and muskoxen show a clear preference for vegetation areas dominated by fen, grassland and salix snow beds, accounting for approximately 75 percent of observations, although these vegetation types only represent approximately 30 percent of the census area (Berg et al. 2008).

\subsection{Sensitivity Analyses}

We consider the sensitivity of the results on both the priors specified on the parameters and the modelling assumptions. We begin by considering the sensitivity of the posterior results on the priors specified. In particular, we increase the prior standard deviation of the Normal priors on the underlying intensity rate, $\theta_{1}$, and interaction parameters, $\theta_{2}$, by a factor of 10 and also specify $\sigma \sim U[0,100]$ (Gelman 2006). The posterior results appear to be insensitive to these different priors, with the same interpretation of the posterior results.

To assess the sensitivity of the model assumptions we consider a range of sensitivity analyses. In particular, we consider (i) the model with an additional temporal random component in the interaction function; (ii) changing the specified interaction radius; and (iii) removing the independence assumption on the random effect terms for the intensity function. We consider each in turn.

\section{(i) Temporal random effects for interaction function}

We consider an additional temporal random effect component on the interaction parameter, analogous to the random effect component on the intensity parameter. In particular, for the observed point process $\boldsymbol{x}_{t}$ for $t=1, \ldots, T$, we set,

$$
\eta_{t}=\exp \left(\theta_{2}+\phi_{2, t}\right),
$$

such that $\phi_{2, t} \sim N\left(0, \nu^{2}\right)$, independently, and where $\nu^{2}$ is a parameter to be estimated. The corresponding likelihood contribution for point process $\boldsymbol{x}_{t}$ is given by,

$$
\begin{aligned}
f\left(\boldsymbol{x}_{t} \mid \boldsymbol{\zeta}, \nu^{2}, \boldsymbol{y}\right)= & \int_{\mathbb{R}} \int_{\mathbb{R}}\left[\alpha\left(\boldsymbol{\zeta}_{t}, \nu^{2}\right) \exp \left(\left[\theta_{2}+\phi_{2, t}\right] \times\left[1-B_{W, t}\left(\boldsymbol{x}_{t}\right)\right]\right)\right. \\
& \left.\times \prod_{j=1}^{N_{t}} \exp \left(\theta_{1}+\phi_{1, t}+\delta \times y\left(x_{t j}\right)\right) p\left(\phi_{1, t} \mid \sigma^{2}\right) p\left(\phi_{2, t} \mid \nu^{2}\right)\right] d \phi_{2, t} d \phi_{1, t},
\end{aligned}
$$


where $p(\cdot \mid \cdot)$ denotes the probability density function of a normal distribution with mean zero and given variance term, evaluated at the specified value, and

$\alpha\left(\boldsymbol{\zeta}_{t}, \nu^{2}\right)=\exp \left(-\int_{W} \exp \left(\left[\theta_{2}+\phi_{2, t}\right] \times\left[1-B_{W, t}(u)\right]\right) \exp \left(\theta_{1}+\phi_{1, t}+\delta \times y(u)\right) d u\right)$.

A data augmentation approach, analogous to that described in Section 4 can be implemented, introducing $\phi_{2}=\left\{\phi_{2, t}: t=1, \ldots, T\right\}$ as auxiliary variables within the Bayesian analysis.

The results obtained for the underlying intensity rate and regression coefficient were generally very similar, with only minor changes in the posterior summary statistics for these parameters. For example, the posterior median $(95 \%$ symmetric CI $)$ for $\theta_{1}$ and $\delta$ were $-14.94(-15.31,-14.51)$ and $-0.64(-0.81$, $-0.48)$, respectively. The estimates of the intensity random effect terms $\left(\sigma^{2}\right.$ and $\phi_{1}$ ) were generally similar (posterior median $(95 \% \mathrm{CI})$ for $\sigma^{2}$ of $0.29(0.10,1.24)$ - the credible interval is slightly larger than for the previous model, which is to be expected since an additional parameter has to be estimated). In particular the interpretation of the random effect terms on the intensity parameters remains consistent, with evidence for a negative random effect in years 1999-2001 and 2003 and a positive random effect in years 2004-7. The underlying interaction parameter $\theta_{2}$ has a significantly increased posterior standard deviation with the addition of temporal heterogeneity on the interaction function. The posterior median (standard deviation) of 0.09 (0.98) and 95\% CI(-2.24, 1.81). The corresponding random effect variance has posterior median $(95 \%$ symmetric CI) of $3.31(0.31,20.73)$. There is only (positive) evidence for three years that the interaction differs to the mean underlying interaction rate, corresponding to a higher interaction in 2007 and lower interaction in years 2004 and 2006. This sensitivity analysis suggests that there is relatively little information contained in the data relating to the interaction among the muskoxen herds, leading to a generally poor precision of the estimation of these parameters.

\section{(ii) Interaction radius}

To assess the dependence of the results on the interaction radius specified, we repeat the analysis of the model specified in Section 3 for two further interaction radii. In particular we consider interaction radii corresponding to changing this radius by a factor of approximately one third (i.e. increasing or decreasing the radius by one third, and rounding to the nearest 10). In particular, we consider an increased interaction radius of 170 , so that muskoxen herds are able to interact with herds further away than previously; and a decreased interaction radius of 80 . We note than interaction radius of 80 leads to two snapshots, corresponding to the years 1999 and 2001, for which no herds were within the interacting distance of each other, i.e. no recorded points within a distance of $2 R$ of each other. (A minimum radius of 112 is needed to ensure all snapshots have herds within the interacting distance). As would be expected, when the interaction radius is decreased, the interaction function provides evidence for a stronger inhibition (posterior median of $\theta_{2}$ of -1.77 with $95 \%$ symmetric CI 
$(-3.83,-0.19)$, with fewer points within the interacting distance of each other. When the interaction radius in increased, there is evidence for a clustering effect (posterior median of $\theta_{2}$ of 1.33 with $95 \%$ symmetric CI $(0.80,1.82)$ due to the number of points within the interacting distance of each other. However, in all analyses, the negative relationship between the intensity and altitude is consistent, with posterior medians for $\delta$ within \pm 0.03 of the previous analysis with $R=125$ and very similar widths for the $95 \%$ credible intervals. The underlying mean intensity rate, $\theta_{1}$, is consistent for the different interaction radii, with the same posterior median to 1 decimal place, and similar width 95\% credible intervals; and the same years identified (2004 and 2007) with the $95 \%$ symmetric CIs for the random effects terms not containing 0 within the intensity function.

\section{(iii) Dependent temporal random effects}

Finally, we remove the independence assumption on the random effect error terms and consider alternative (non-independent) random effect models: a random walk and a moving average of order 1 (i.e. an $M A(1)$ model). For example, the random walk process is specified such that for $t=2, \ldots, T$,

$$
\phi_{1, t} \sim N\left(\phi_{1, t-1}, \sigma_{R W}^{2}\right)
$$

where $\sigma_{R W}^{2}$ is the associated random walk variance to be estimated. For the $M A(1)$ model for $t=2, \ldots, T$, we specify,

$$
\phi_{1, t}=k \omega_{t-1}+\omega_{t}
$$

such that $\omega_{s} \sim N\left(0, \sigma_{M A}^{2}\right)$, independently for all $s=1, \ldots, T$. For identifiability for both models we specify $\phi_{1,1}=0$. We specify independent (non-informative) priors, such that $\sigma_{R W}^{2}, \sigma_{M A}^{2} \sim \Gamma^{-1}(0.001,0.001)$ and $k \sim N\left(0,100^{2}\right)$ for the $A R(1)$ and $M A(1)$ models.

For each of these models, very similar results are obtained for the environmental regression coefficient, $\delta$, intensity parameters for each year, $\left(\theta_{1}+\phi_{1, t}\right)$ for $t=1, \ldots, T$ and interaction parameter, $\theta_{2}$ (i.e. the same posterior median and $95 \%$ CIs to 2 significant figures). For the $M A(1)$ process, the posterior median $(95 \% \mathrm{CI})$ for $\sigma_{M A}^{2}$ is $0.17(0.05,0.71)$, and for $k, 0.37(-0.18,0.82)$. The posterior credible interval for the variance component significantly overlaps with that obtained assuming independent random effects (see Table 2). In particular, the probability that the random effect variance for the $M A(1)$ model is lower than that for the independent model, $\mathbb{P}_{\pi}\left(\sigma_{M A}^{2}<\sigma^{2}\right.$ ), is 0.68 (or Bayes factor of $2.16)$, which does not provide positive support for a lower variance component. In addition, the value of $k=0$, corresponding to independent random effects is also contained within the $95 \%$ CI for this parameter. For the random walk the posterior median $(95 \% \mathrm{CI})$ for the random effect variance component, $\sigma_{R W}^{2}$, is $0.14(0.04,0.63)$. Once more the posterior credible interval for the variance component significantly overlaps with that obtained when the random effects terms are independent (and when an $M A(1)$ model is fitted). The posterior 
probability that the variance component is less for the random walk model than the independent model, $\mathbb{P}_{\pi}\left(\sigma_{R W}^{2}<\sigma^{2}\right)$, is 0.73 (or Bayes factor of 2.81), close to positive evidence of a smaller variance term (Kass and Raftery 1995). However, we note that with only 9 snapshots within our data series, the ability to identifying additional structure within the random effects will be limited.

Overall, the similarity in the results and corresponding interpretation for the intensity and regression coefficient parameters across the different models and priors suggests that these results are generally robust with regard to the model specified on the interaction function.

\section{Discussion}

Collecting ecological data on population dynamics and spatial distribution of long lived animals is associated with a number of logistic and often also financial challenges. Data series following a certain population of individually marked animals over long time whilst sampling a wide range of biotic and abiotic parameters, such as the Soya sheep data from St Kilda (Clutton-Brock and Pemberton 2004) are of high value in ecology, but uncommon. Fragmented and/or sparse data sets are common in biology, and may pose a limit to the ecological questions that can be addressed. Nevertheless, understanding the temporal and/or spatial dynamics of populations is of fundamental importance for species management and conservation (Aarts et al. 2008). The spatial position of animals is of paramount importance in understanding the interactions of individuals, populations and species with their environment. Methods which can aid transferring of data from data-poor to data-rich areas or utilise repeated measures more efficiently are therefore potentially relevant within a wide range of biological applications.

We have presented a robust integrated approach for analysing a series of spatial point patterns, where a single individual pattern does not in itself provide sufficient information to realistically incorporate or allow the interpretation of interesting biological processes. However, analysing the sequential point patterns simultaneously, allowing for temporal heterogeneity within the intensity function, we are able to decode some of the spatial relationship between a large ungulate and spatial covariate (altitude) whilst permitting social interactions between herds. The temporal biological model is formulated mathematically by introducing a random effects component (on the intensity function) within the model structure. This approach uses all the available information when fitting the model to the data, so that more precise and consistent estimates for datasets with relatively few data points (and hence limited information in the data) can be obtained, by essentially "borrowing" information from the other independent datasets and providing consistent estimates across all the spatial point patterns. Consequently, we demonstrate that implementing a single integrated analysis can significantly increase the precision of the parameter estimates when there is limited information on a particular parameter(s), for example, the interaction 
parameter for the study dataset. Using this approach for the muskoxen data there appears to be evidence for a strong negative relationship between the intensity of the muskoxen and the altitude, so that muskoxen appear to prefer the lower regions of the study area, previously identified by Forchhammer et al. (2005). This relationship is less clear within the single independent analyses. For the given dataset, we primarily considered a random effect component on the underlying intensity parameter, but the approach is generalisable, for example, to allow for temporal heterogeneity on the regression coefficient for the environmental covariate. Since interaction concerns second and higher order information in the data there is typically less information contained within the data relating to the interaction function than the intensity function. In particular in the context of datasets with relatively few observed points, this would also suggest that it may be difficult to identify temporal heterogeneity within the interaction parameter, as is the case here when a random effect component was added.

We have described and implemented a Bayesian data augmentation approach, whereby the temporal random effect terms are imputed within the MCMC algorithm and essentially integrated out by considering the marginal distribution of the model parameters. Consequently, since the random effect terms are imputed at each iteration of the Markov chain, obtaining posterior estimates or these random effect terms is immediate. These can be of interest in themselves, for example, in identifying which datasets have a positive/negative temporal random effect component within the intensity function and/or comparing the magnitude of the random effects between datasets to investigate the strongest deviation from the underlying mean. In addition, and in contrast to the previous approach proposed by Illian and Hendrichsen (2010), we are able to easily obtain posterior credible intervals for each of the parameters providing a measure of the precision of the parameters of interest.

Future work in this area includes the consideration of model selection in terms of covariates that may be present in the model. In particular, it is currently not known what drives the variation in population size and influx into Zackenberg during the summer months, but the change in population density coincides with a marked advancement in the onset of spring affecting the phenology of a wide range of species in the Zackenberg region. Thus additional covariates that we may wish to assess for a relationship with intensity (or interaction) include vegetation composition, normalised difference vegetation index (NDVI) and spring snow cover. Model selection within the classical framework can be achieved via the use of likelihood ratio tests and/or AIC statistic. Alternatively, within the Bayesian framework Bayes Factors (or equivalently, posterior model probabilities) can be used to quantitatively discriminate between the competing models, and calculated using, for example, reversible jump Markov chain Monte Carlo (Green 1995). In the analyses within this paper, the posterior results are largely insensitive to the prior specification on the parameters. However, Bayes Factors (by their mathematical definition) are typically sensitive to the priors specified on these model parameters. Thus, further research includes the sensitivity of the Bayes Factors on the priors specified on the parameters 
and consequently the specification of biologically plausible priors. Applying such an approach would permit a detailed analyses of the joint factors of social interactions and resource availability which both shape animal spatial distributions. Such analyses therefore may provide an improved use of sparse data to explain ecological patterns. In addition, much recent research has focused on developing computationally efficient methods for fitting spatial models in a Bayesian context based on integrated nested Laplace approximations (INLA) (Rue et al. 2009); see also the work by Lindgren et al. (2011). For example, a log Gaussian Cox process model has been fitted to replicated patterns formed by the muskoxen herds (Illian et al 2012a,b), although this approach cannot account for second or higher order spatial behaviour. The application of the INLA approach to such spatial point processes is an area of ongoing research.

\section{Acknowledgements}

We would like to thank the Zackenberg Basic programmes who collected the muskoxen data and made the data available to us, with particular thanks to Niels Martin Schmidt. In addition we would like to thank Brett McClintock and Roland Langrock for some useful conversations. The authors also gratefully acknowledge the financial support of Research Councils UK for Illian.

\section{References}

Aarts, G., MacKenzie, M., McConnell, B., Fedak, M. and Matthiopoulos, J. (2008), Estimating space-use and habitat preference from wildlife telemetry data. Ecography 31, 140-160

Babin, J. -S., Fortin, D., Wilmshurst, J. F. and Fortin, M. -E. (2011), Energy gains predict the distribution of plains bison across populations and ecosystems. Ecology 92, 240-252

Baddeley, A. J. and Turner, T. R. (2000), Practical maximum pseudolikelihood for spatial point patterns (with discussion). Australian and New Zealand Journal of Statistics 32, 283-322

Baddeley, A. J. and Turner, T. R. (2006), Modelling spatial point patterns in R. In A. Baddeley, P. Gregori, J. Mateu, R. Stoica, and D. Stoyan, (eds.), Case Studies in Spatial Point Pattern Modelling, number 185 in Lecture Notes in Statistics, 23-74. Springer-Verlag, New York

Baddeley, A. J. and Turner, T. R. (2011), spatstat website. URL: www.spatstat.org.

Baddeley, A. J. and van Lieshout, M. N. W. (1995), Area-interaction point processes. Annals of the Instituts of Statistical Mathematics 47, 601-619

Berg, T. B. (2003), Mammals. In Rasch, M. and Canning, K. (eds), Zackenberg Ecological Research Operations. 8th Annual Report. Danish Polar Center, Copenhagen, Denmark, pp 43-50 
Berg, T. B, Schmidt, N. M., Høye, T. T., Aastrup, P., Hendrichsen, D. K., Forchhammer, M. C. and Klein, D. R. (2008), High-arctic plant-herbivore interactions under climate influence. Advances in Ecological Research 40, 275-298

Berman, M. and Turner, M. R. (1992), Approximation point process likelihoods with GLIM. Journal of the Royal Statistical Society, Series C 41, $31-38$

Besbeas, P., Borysiewicz, R. S. and Morgan, B. J. T. (2008), Completing the ecological jigsaw. In D. L. Thomson, E. G. Cooch and M. J. Conroy (eds.), Modeling Demographic Processes in Marked Populations, Springer - Series: Environmental and Ecological Statistics, Volume 3, pp. 513-540

Besag, J. (1978), Some methods of statistical analysis for spatial data. Bulletin of the International Statistical Institute 47, 77-92.

Besbeas, P., Lebreton, J. D. and Morgan, B. J. T. (2003), The efficient integration of abundance and demographic data. Journal of the Royal Statistical Society, Series C 52, 95-102

Blackwell, P. G. (2003), Bayesian inference for Markov processes with diffusion and discrete components. Biometrika 90, 613-627

Bonner, S. J., Morgan, B. J. T. and King, R. (2010), Continuous covariates in mark-recapture-recovery analysis: A comparison of methods. Biometrics 66, 1256-1265

Breed, G. A., Jonsen, I. D., Myers, W. D., Bowen, W. D. and Leonard, M. L. (2009), Sex-specific, seasonal foraging tactics of adult grey seals (Halichoerus grypus) revealed by state-space analysis. Ecology 90, 32093221

Brooks, S. P. (1998), Markov chain Monte Carlo method and its application. The Statistician 47, 69-100

Brooks, S. P. and Gelman, A. (1998), Alternative methods for monitoring convergence of iterative simulations. Journal of Computational and Graphical Statistics 7, 434-455

Brooks, S. P., King, R. and Morgan, B. J. T. (2004), A Bayesian approach to combining animal abundance and demographic data. Animal Biodiversity and Conservation 27, 515-529

Brooker, R. W., Maestre, F. T., Callaway, R. M., Lortie, C. L., Cavieres, L. A., Kunstler, G., Liancourt, P., Tielborger, K., Travis, J. M. J., Anthelme, F., Armas, C., Coll, L., Corcket, E., Delzon, S., Forey, E., Kikvidze, Z., Olofsson, J., Pugnaire, F., Quiroz, Q. L., Saccone, P., Schiffers, K., Seifan, M., Touzard, B. and Michalet, R. (2008), Facilitation in plant communities: the past, the present, and the future. Journal of Ecology 96, 18-34

Caraco, T., Martindale, S., Pulliam, H. R. (1980), Flocking: advantages and disadvantages. Nature 285, 400-401 
Cave, V. M., King, R. and Freeman, S. N. (2010), An integrated population model from constant effort bird-ringing data. Journal of Agricultural, Biological, and Environmental Statistics 15, 119-137

Clutton-Brock, T. H. and Pemberton J. (2004), Soay Sheep, Cambridge University Press.

Cornulier T. and Bretagnolle V. (2006), Assessing the influence of environmental heterogeneity on bird spacing patterns: a case study with two raptors. Ecography 29, 240-250

Elberling, B., Tamstorf, M. P., Michelsen, A., Arndal, M. F., Sigsgaard, C., Illeris, L., Bay, C., Hansen, B. U., Christensen, T. R., Hansen, E. S., Jakobsen, B. H. and Beyens, L. (2008), Soil and plant community-characteristics and dynamics at Zackenberg. Advances in Ecological Research 40, 223-248

Forchhammer, M. C., Post, E., Berg, T. B., Høye, T. T. and Schmidt, N. M. (2005), Local-scale and short-term herbivore-plant spatial dynamics reflect influences of large-scale climate. Ecology 86, 2644-2651

Forchhammer, M. C., Schmidt, N. M., Høye, T. T., Berg, T. B., Hendrichsen, D. K. and Post, E. (2008), Population dynamical responses to climate change. Advances in Ecological Research 40, 391-419

Gauthier, G., Besbeas, P., Lebreton, J. D. and Morgan, B. J. T. (2007), Population growth in greater snow geese: a modeling approach to integrating demographic and population survey information. Ecology 88, 1420-1429

Gelman, A. (2006), Prior distributions for variance parameters in hierarchical models. Bayesian Analysis 1, 515-534

Green, P. J. (1995), Reversible jump Markov chain Monte Carlo computation and Bayesian model determination. Biometrika 82, 711-732

Hansen J., Hansen, L. H., Christoffersen, K. S., Albert, K. R., Skovgaard, M. S., Bay, C., Kristensen, D. K., Berg, T. B., Lund, M., BoulangerLapointe, N., Sørensen, P. L., Christensen, M. U. and Schmidt, N. M. (2011), Zackenberg Basic: The BioBasis programme. In Jensen, L. M. and Rasch, M. (eds.), Zackenberg Ecological Research Operations, 16th Annual Report, 2010. Aarhus University, DCE Danish Centre for Environment and Energy, 2011. pp. 43-66

Hassell, M. P. (1975), Density-dependence in single-species populations. Journal of Animal Ecology 44, 283-295

Illera, J. C., von Wehrden, H. and Wehner, J. (2010). Nest site selection and the effects of land use in a multi-scale approach on the distribution of a passerine in an island arid environment. Journal of Arid Environments 74, 1408-1412

Illian, J. B. and Hendrichsen, D. K. (2010). Gibbs point process models with mixed effects. Environmetrics 21, 341-353 
Illian, J. B., Møller, J. and Waagepetersen, R. P. (2009). Hierarchical spatial point process analysis for a plant community with high biodiversity. Environmental and Ecological Statistics 16, 389-405

Illian, J. B., Penttinen, A., Stoyan, H. and Stoyan, D. (2008). Statistical Analysis and Modelling of Spatial Point Patterns. John Wiley and Sons, Chichester

Illian, J. B., Sørbye, S. H. and Rue, H. (2012a). A toolbox for fitting complex spatial point process models using integrated Laplace transformation (INLA). Annals of Applied Statistics - in press

Illian, J. B., Sørbye, S. H., Rue, H. and Hendrichsen, D. (2012b). Using INLA to fit a complex point process model with temporally varying effects - a Case study Journal of Enviromental Statistics - in press

Janson, C. H. (1988), Food competition in brown capuchin monkeys (Cebus apella): quantitative effects of group size and tree productivity. Behaviour 105, 53-76

Jetz, W., Carbone, C., Fulford, J. and Brown, J. H. (2004), The Scaling of Animal Space Use. Science 306 266-268

Jonsen, I. D., Flemming, J. M. and Myers, R. A. (2005), Robust state-space modeling of animal movement data. Ecology 86, 2874-2880

Johnson, D. S., London, J. M., Lea M. -A. and Durban, J. W. (2008), Continuous-time correlated random walk model for animal telemetry data. Ecology 89, 1208-1215

Kass, R. E. and Raftery, A. E. (1995), Bayes factors. Journal of the American Statistical Association 90, 773-793

King, R., Brooks, S. P., Mazzetta, C., Freeman, S. N. and Morgan, B. J. T. (2008), Identifying and diagnosing population declines: A Bayesian assessment of lapwings in the UK. Journal of the Royal Statistical Society, Series C 57, 609-632

King, R., Morgan, B. J. T., Gimenez, O. and Brooks, S. P. (2009), Bayesian Analysis for Population Ecology. CRC Press, Boca Raton

Langrock, R., King, R., Matthiopulos, J., Thomas, L., Fortin, D. and Morales, J. M. (2012), Flexible and practical modeling of animal telemetry data: hidden Markov models and extensions. Under revision for Ecology (Statistical Reports)

Law, R., Purves, D., Murrell, D., and Dieckmann, U. (2001). Causes and effects of small scale spatial structure in plant populations. In J. Silvertown and J. Antonovics (Eds.), Integrating Ecology and Evolution in a spatial context, Blackwell Science, Oxford, pp. 21-44

Li, C., Jiang, Z. G., Li, L. L., Li, Z. Q., Fang, H. X., Li, C. W. and Bauchamp, G. (2012), Effects of reproductive status, social rank, sex and group size on vigilance patterns in Przewalski's gazelle. PlosOne 7, e32607 DOI: 10.1371/journal.pone. 0032607 
Lindgren, F., Lindström, J. and Rue, H. (2011) An explicit link between Gaussian fields and Gaussian Markov random fields: the stochastic partial differential equation approach (with discussion). Journal of the Royal Statistical Society, Series B 73, 423-498

McClintock, B. T., King, R., Thomas, L., Matthiopulos, J., McConnell, B. J. and Morales, J. M. (2012) A general discrete-time modeling framework for animal movement using multi-state random walks. Ecological Monographs in press

McCrea, R. S., Morgan, B. J. T., Gimenez, O., Besbeas, P., Lebreton, J. D. and Bregnballe, T. (2010), Multi-site integrated population modelling. Journal of Agricultural, Biological, and Environmental Statistics 15, 539538

Møller, J. and Waagepetersen, R. P. (2007), Modern statistics for spatial point processes (with discussion). Scandinavian Journal of Statistics 34, $643-711$

Morales, J. M., Haydon, D. T., Frair J., Holsinger, K. E. and Fryxell, J. M. (2004), Extracting more out of relocation data: building movement models as mixtures of random walks. Ecology 89, 2436-2445

Nicholson, A. J. (1954), An outline of the dynamics of animal populations. Austalian Journal of Zoology 2, 9-65

Reynolds, T. J., King, R., Harwood, J., Frederikesen, M., Harris, M. P. and Wanless, S. (2009), Integrated data analyses in the presence of emigration and tag-loss. Journal of Agricultural, Biological, and Environmental Statistics 14, 411-431

Rue, H., Martino, S., and Chopin, N. (2009), Approximate Bayesian inference for latent Gaussian models using integrated nested Laplace approximations (with discussion). Journal of the Royal Statistical Society, Series B 71, 319-392

Schaub, M., Gimenez, O., Sierro, S., and Arlettaz, R. (2007), Assessing population dynamics from limited data with integrated modeling: life history of the endangered greater horseshoe bat. Conservation Biology 21, 945-955.

Stoyan, D., Kendall, W. and Mecke, J. (1995), Stochastic Geometry and its Applications (2nd edn). John Wiley and Sons, London

Tanner, M. A. and Wong, W. H. (1987), The calculation of posterior distribution by data augmentation. Journal of the American Statistical Association 82, 528-540

van Lieshout, M. N. M. (2000), Markov Point Processes and their Applications. Imperial College Press, London

Wikelski M., Moxley J., Eaton-Mordas A., López-Uribe M. M., Holland R., Moskowitz, D., Roubik, D. W. and Kays, R. (2010), Large-Range Movements of Neotropical Orchid Bees Observed via Radio Telemetry. PLoS ONE 5, e10738 
Figure 1: The location of the muskoxen in each snapshot for years 1999-2007 and corresponding contours of the altitude, with the boundary of the study area included.

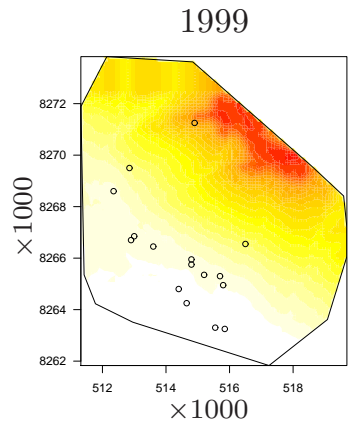

(a)

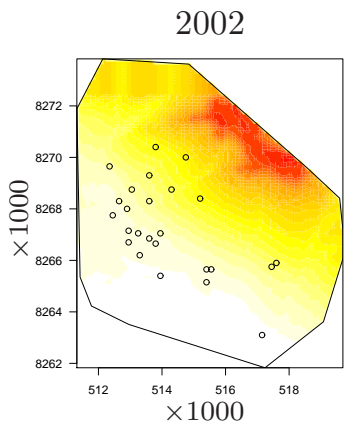

(d)

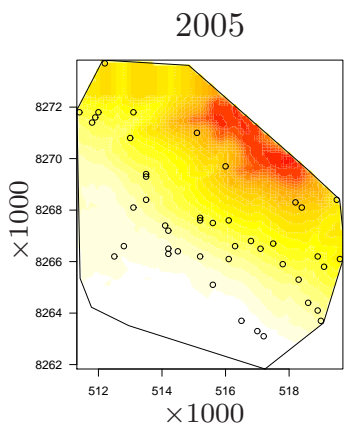

(g)

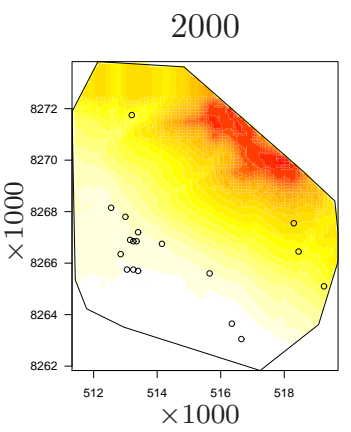

(b)

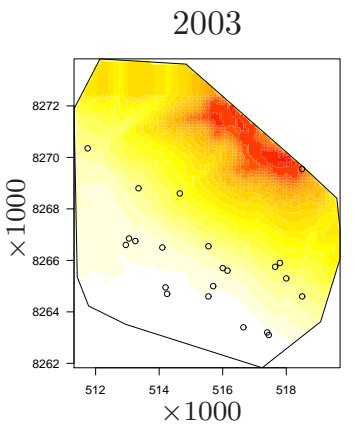

(e)

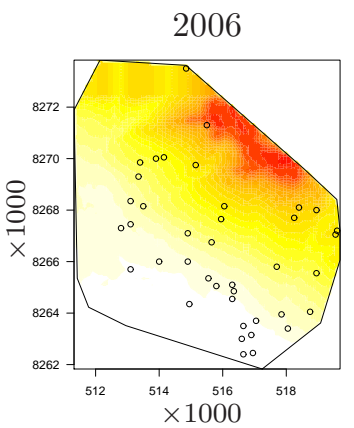

(h)

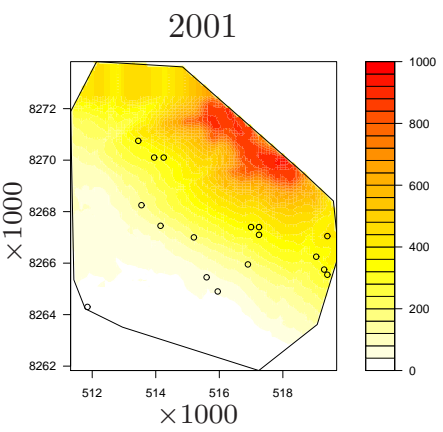

(c)

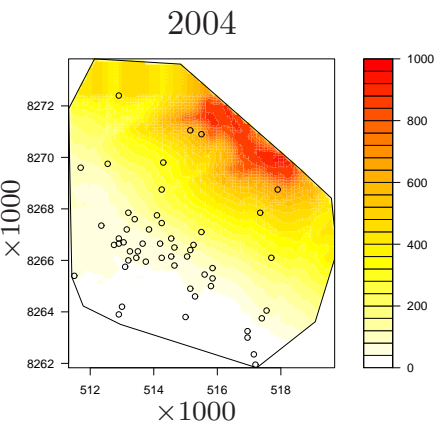

(f)

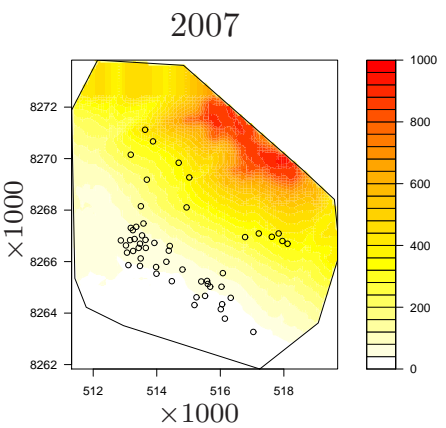

(i) 
Table 1: Independent analyses for each spatial point pattern for years 1999-2007, using both classical and Bayesian approaches.

(a) MLE for each parameter

\begin{tabular}{c|c|c|c} 
& $\theta_{1, t}$ & $\theta_{2, t}$ & $\delta_{t}$ \\
Year & MLE & MLE & MLE \\
\hline 1999 & -15.93 & 0.29 & -1.31 \\
2000 & -15.81 & 3.29 & -0.98 \\
2001 & -15.24 & -5.06 & -0.11 \\
2002 & -15.19 & -0.53 & -0.89 \\
2003 & -15.37 & 1.60 & -0.82 \\
2004 & -14.40 & -2.38 & -1.00 \\
2005 & -14.37 & 0.69 & -0.19 \\
2006 & -14.43 & -3.37 & -0.41 \\
2007 & -14.60 & 1.20 & -0.97
\end{tabular}

(b) Posterior median (standard deviation) and 95\% symmetric credible interval (CI) for each parameter

\begin{tabular}{c|cc|cc|cc} 
& \multicolumn{2}{|c|}{$\theta_{1}$} & \multicolumn{2}{c|}{$\theta_{2}$} & \multicolumn{2}{c}{$\delta$} \\
Year & Median (SD) & $95 \%$ CI & Median (SD) & $95 \%$ CI & Median (SD) & $95 \%$ CI \\
\hline 1999 & $-16.07(0.50)$ & $(-17.26,-15.28)$ & $-0.47(2.70)$ & $(-7.66,3.04)$ & $-1.42(0.56)$ & $(-2.73,-0.52)$ \\
2000 & $-15.92(0.40)$ & $(-16.84,-15.26)$ & $3.19(0.98)$ & $(0.89,4.70)$ & $-1.05(0.44)$ & $(-2.08,-0.35)$ \\
2001 & $-15.29(0.26)$ & $(-15.84,-14.83)$ & $-10.33(12.78)$ & $(-46.33,1.07)$ & $-0.13(0.26)$ & $(-0.69,0.34)$ \\
2002 & $-15.24(0.29)$ & $(-15.89,-14.73)$ & $-1.03(2.25)$ & $(-6.71,2.10)$ & $-0.92(0.33)$ & $(-1.66,-0.36)$ \\
2003 & $-15.44(0.31)$ & $(-16.15,-14.90)$ & $1.44(1.39)$ & $(-1.92,3.54)$ & $-0.87(0.35)$ & $(-1.66,-0.29)$ \\
2004 & $-14.43(0.20)$ & $(-14.88,-14.08)$ & $-2.59(1.64)$ & $(-6.62,-0.18)$ & $-1.02(0.24)$ & $(-1.54,-0.61)$ \\
2005 & $-14.39(0.17)$ & $(-14.73,-14.08)$ & $0.54(1.05)$ & $(-1.89,2.52)$ & $-0.20(0.16)$ & $(-0.55,0.10)$ \\
2006 & $-14.45(0.17)$ & $(-14.81,-14.12)$ & $-4.24(3.20)$ & $(-12.49,0.06)$ & $-0.41(0.19)$ & $(-0.82,-0.07)$ \\
2007 & $-14.63(0.21)$ & $(-15.08,-14.27)$ & $1.18(0.63)$ & $(-0.26,2.23)$ & $-0.99(0.24)$ & $(-1.50,-0.57)$
\end{tabular}


Table 2: Posterior median (standard deviation) and 95\% symmetric credible interval (CI) for each parameter in the integrated analysis.

\begin{tabular}{c|cc} 
& Posterior & \\
& median (SD) & $95 \%$ CI \\
\hline$\theta_{1}$ & $-14.94(0.20)$ & $(-15.32,-14.53)$ \\
$\sigma^{2}$ & $0.27(0.42)$ & $(0.09,1.06)$ \\
$\delta$ & $-0.65(0.09)$ & $(-0.82,-0.49)$ \\
\hline$\theta_{2}$ & $0.46(0.39)$ & $(-0.35,1.18)$
\end{tabular}

Random effects - $\phi_{1, t}$

\begin{tabular}{c|cc} 
Year & $\begin{array}{c}\text { Posterior } \\
\text { median (SD) }\end{array}$ & $95 \%$ CI \\
\hline 1999 & $-0.49(0.27)$ & $(-1.06,-0.01)$ \\
2000 & $-0.40(0.26)$ & $(-0.97,0.08)$ \\
2001 & $-0.44(0.28)$ & $(-1.03,0.06)$ \\
2002 & $-0.14(0.25)$ & $(-0.67,0.35)$ \\
2003 & $-0.25(0.25)$ & $(-0.76,0.23)$ \\
2004 & $0.55(0.23)$ & $(0.10,1.00)$ \\
2005 & $0.38(0.23)$ & $(-0.07,0.83)$ \\
2006 & $0.26(0.23)$ & $(-0.20,0.73)$ \\
2007 & $0.52(0.23)$ & $(0.08,0.98)$
\end{tabular}

